\section{ATSCALE: An evaluation of unidimensionality and internal consistency of responses to a series of questions*}

\author{
BARBARA A. McGARY and JOHN A. BURNS \\ Northwestern University, Evanston, Illinois 60201
}

When a person's response to a number of questions is summarized by a single score and whenever that score is given a content-referent name, it is assumed that all questions are tapping a single common trait, attitude, or factor. The ATSCALE program provides a means of checking this assumption by evaluating thr single-factoredness or unidimensionality of a scale $:$, well as its internal consistency. This program combines the major features of two programs which have been previously reported (Morrison, Campbell, \& Wolins, 1967; Werner, 1967), but uses less core storage and time than either of the original programs.

\section{INPUT}

The data input for analysis consists of one record for each individual containing the mul level responses to a series of questions. Response frequ ncies are tallied for each item, and an intercorrelation matrix is presented which provides for correlation of items and total scores (scored in the Likert tradition) with up to seven external criteria. Multilevel responses are dichotomized by the program employing cutoff points indicated by the user.

\section{OUTPUT}

Statistics are presented in the program which purport to measure reproducibility or homogeneity of dichotomous responses: (a) Guttman's coefficient of reproducibility; (b) chance, obtained, and ideal expectancies of scale types; (c) Green B, and Green Index of Consistency (B); (d) Loevinger Index of Homogeneity; (e) Kuder-Richardson Formula 20, corrected $\mathrm{K}-\mathrm{R} 20$. Other output based on the multilevel responses include: (a) Cronbach's alpha (Hoyt analysis of variance); (b) inferred average interitem correlations; (c) two item-total correlations. In addition, two methods of extracting a single factor are presented as well as an index of the extent to which a single factor is present.

\section{LIMITATIONS}

This FORTRAN program will accommodate a scale with a maximum of 30 items at a time, chosen for analysis from a maximum of 200 items. Each item may have as many as 10 levels. The number of respondents is limited to 350 . The core required to run this program on the CDC 6400 is 43,000 locations.

\section{AV AILABILITY}

A copy of the source deck, a listing and a description of the control card input, is available from Northwestern University's Computer Center, Evanston, Illinois 60201, as Program NUCC281. A version with a larger capacity, a description of the ATSCALE program logic, and a manual which provides guidelines for the interpretation of statistics included in the program are also available upon request.

\section{REFERENCES}

Morrison, D. G., Campbell, D. T., \& Wolins, L. A Fortran program for evaluating internal consistency and single-factoredness in sets of multi-level attitude items. A T T A NAL NUCC0048, Vogelback Computer Center, Northwestern University, 1965 (revised 1967).

Werner, R. A. Fortran program for Guttman and other scalogram analyses. GUTTSCL NUCC0115, Vogelback Computer Center, Northwestern University, 1965 (revised 1967).

\section{SPRAY: A CDC6600 FORTRAN IV program}

\author{
JOSEPH K. UNEKIS \\ Indiana University, Bloomington, Indiana 47401
}

This program is designed to recode information that has been multipunched or recorded in a binary form.

By far the most popular way to record information onto punched cards is to use the Hollerith coding system. With the Hollerith code, one piece of information (a letter, digit, or special character) is represented by a unique combination of punches in a single column of a card. Because of its popularity, almost all computer installations use it as the standard method of reading information into the computer. There are alternative methods of representing information, however, and these methods sometimes cause the researcher difficulty when he or she attempts to utilize the data in a normal computer environment.

First, some coding schemes are designed to take advantage of the availability of 12 rows in a card column. By assigning each row a unique meaning, the answers to several questions on an interview schedule can be coded into one column. For example, the first 3 rows might stand for the respondent's sex $(12=\mathrm{M}, 11=$ $\mathrm{F}, 0=\mathrm{NA})$, the next 4 rows his race $(1=\mathrm{W}, 2=\mathrm{B}, 3=$ other, $4=\mathrm{NA})$, and the last 5 rows his education ( $5=$ GS, $6=\mathrm{HS}, 7=$ Coll, $8=$ other, $9=\mathrm{NA}) . \mathrm{A}$ college-educated white male would be coded 12-1-7 in a single column. However, an attempt to read this column on most card readers would end in failure because 12-1-7 is not a valid Hollerith punch.

Second, some early studies (especially the Michigan SRC studies) utilized the 12-row feature of punched cards in another manner. By using all 12 legitimate single Hollerith representations, they were able to code 12 separate categories in each column. Since there are only 10 digits in the decimal system, the two special characters + and - were used. This scheme works fine when categories are tabulated on counter sorters, but it creates problems when computers are used to analyze the data.

Third, some archives (among them the Roper Center for Public Opinion Research) record their information in a binary format when copying to tape. All 12 rows of a card column are represented in their original form on the tape $(0 \mathrm{~s}$ stand for blank rows and $1 \mathrm{~s}$ stand for punched rows). The number three would be represented as 000001000000 , while the letter A would be represented as 100100000000 . In order for this information to be read correctly by a tape driver, it first has to be recoded into either a 6-bit or an 8-bit configuration (depending upon the computer). Since this is not the case, the only alternative is to punch the information to cards and then read them in through the card reader. 